\title{
Status Investigation and Problem Analysis on Graduation Project (thesis) of Colleges and Universities
}

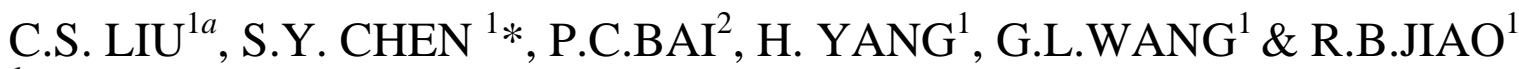 \\ ${ }^{1}$ College of Mechanical Engineering, Jiamusi University, Jiamusi 154007, China \\ ${ }^{2}$ Harbin Dongan Automotive Engine Manufacturing Co., Ltd, Haerbin, 150069, China \\ aliuchunshan_2001@163.com; *chensiyu516@163.com (Corresponding Author)
}

\begin{abstract}
As the last part of the undergraduate education, graduation project (thesis) is greatly affected by changes in social environment, industry situation and policy state. In order to investigate the status of undergraduate graduation project (thesis), 322 undergraduate student who enrolled in Jiamusi University in the year 2009 and 2010 and have various professions are chosen as the subject, and a questionnaire survey on four essential factor which include topic, instructor, attitude of the student and relevant time node is conducted. The results show that: there are many problems existing in some undergraduate graduation project (thesis) needed to be solved as soon as feasible, and reasonable measures concerning all these problems are presented in this paper.
\end{abstract}

KEYWORD: Graduation Project (Thesis); status; questionnaire survey; Problem Analysis

\section{INTRODUCTION}

As an important educational part of undergraduate education in colleges and universities, graduation project (thesis) is a crucial component of talent training project and has a significant effect on qualified and applied personal cultivation. Graduation project (thesis) is not only a process regarding comprehensive utilization of professional knowledge for the students, but also a stage concerning the combination of theory and practice to analyze and solve practical problems and to develop a preliminary scientific research ability. Similarly it is also a general test on the comprehensive quality and engineering practice ability of the students. At the same time, as a practice part, the role and status of graduation project in undergraduate education is irreplaceable for other educational parts. Therefore it becomes a valuable subject to improve and enhance this part and increase the quality of undergraduate education. In recent years, the increasing enrollment and the consolidation of some institutions has directly result in an decrease in the quality of enrolled students. Plagiarism and patchwork together with nonstandard thesis are no longer individual phenomenon. When facing the dilemma about consulting instructor and participating employment interview, most students prefers the latter. Above phenomenon shows that it is one of the important tasks for colleges and universities to improve the quality of graduation project (thesis), and should be taken seriously[2]. Thus, 322 undergraduate student who enrolled in Jiamusi University in the year 2009 and 2010 and have various professions are chosen as the subject, and a questionnaire survey is conducted before graduate. The details of the survey involves four essential factor including topic, instructor, attitude of the student and relevant time node[3].

\section{BASIC INFORMATION SURVEYS}

The survey takes a form of questionnaire survey. Questionnaires are issued to the undergraduate students of Jiamusi University, School of Mechanical Engineering. Professions include Agricultural Engineering, Packaging Engineering, Agricultural Electrification and Automation. The questionnaire issued is 332 , and 330 valid are retrieved. Contents of the questionnaire is divided into four blocks: topic, instructor, attitude of the student and relevant time. The time period lasts from the accomplish of undergraduate reply until the students leave the university.

\section{SURVEY ANALYSIS}

\subsection{Topic}

Strict selection of graduation topics is the base of improving the quality of graduation project. The 
topic of thesis of Agricultural Engineering, Packaging Engineering, Agricultural Electrification and Automation must meet the requirement of the professional training objectives, combine with production practice and scientific research possibly. Thus can help the students to consolidate, deepen and extend what they have learned; Meanwhile, the topic should properly reflect the development level and the leading trend of the industry, embody current technological development grade, reflect the characteristics of the times, update topics continuously to let students standing on the leading position.

\section{a .topic sources}

Graduate project topics comes from sources generally including instructor's research projects, production practices project and student self-issued project. Among these, $21.8 \%$ of the project are from the production practices, which avails the combination of theory and practice; $51.4 \%$ are instructor's research projects, and the study follow the sequence of summarizing the scientific problems by instructors and then working out by students. But instructors should make it clear that students understand the engineering background and how the problem is summarized from the project, otherwise, the student will break away from production practice. The additional $26.8 \%$ are student selfissued project. Students can self issue topics relevant to their own profession during practice in some company. Thus the students can get into character soon after graduate and meet the requirement of work. According to the survey, the sources of graduate project topics are shown in Fig.1.



Fig.1 sources of graduate project

\section{b. topic types}

From the contents and methods, the graduate project (thesis) of agricultural engineering, packaging engineering and agricultural electrification and automation is divided into theoretical research, applied research, software development, and integration etc. according to the survey, the type of the graduate project is shown in Fig.2. The graduate project (thesis) of agricultural engineering, packaging engineering and agricultural electrification and automation is primarily of the theoretical research type, reaching $65.1 \%$. But in practice, single and similar topics are of majority. There are also applied research topics which accounted for $11.5 \%$, software development topics which accounted for $11.5 \%$ and comprehensive graduate topics which accounted for $15 \%$.

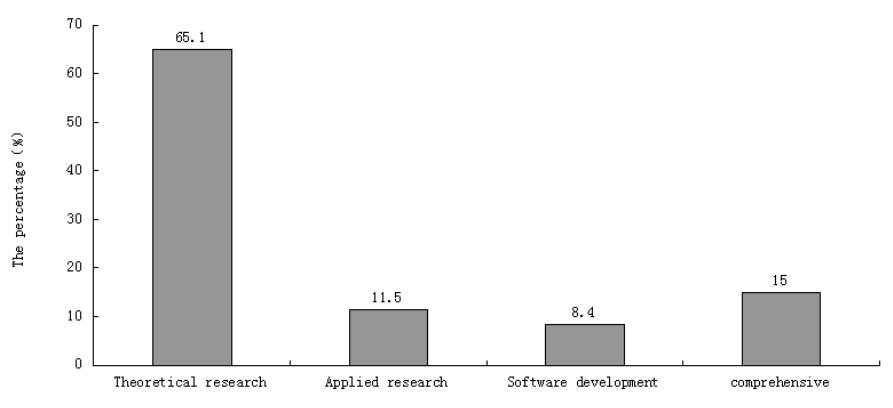

Fig. 2 types of graduate project

\section{c. topic difficulty}

Graduate project requirements for medium topics depth, breadth and difficulty for the students to accomplish the task successfully. Fig. 3 shows the results of the survey on topic difficulty, it can be found that $76.8 \%$ of the students thought the difficulty of the subject is appropriate, can be completed on time. However, some topics are lack of innovation, and are lack of comprehensive training of students, which limits the express of the initiative of students, and ignores the cultivate of the innovation awareness and practical ability. Some topics are too wide, and the students can only generalize; while some topics are too narrow making the design work obviously inadequate; Some topics are too old, lack of comprehensiveness, novelty, depth and breadth; some topics are too difficult for undergraduates, thus less expected results can be received.

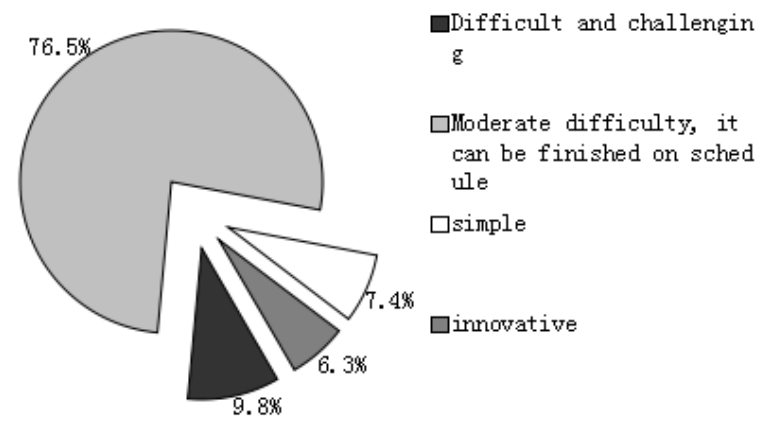

Fig.3 Difficulty on topic selection

\subsection{Instructors}

a. the instructors and the means of communication

For different types of projects, the instructor can take different ways to guidance students different. 
Application type topics, mainly take the form of face to face guidance in the design room, while theoretical issues mainly take the form of telephone, e-mail, QQ and other network communication mode, or on sit communicate guidance. Guidance and communication mode are shown in Fig. $4,73.4 \%$ are by face to face guidance, $8.4 \%$ by the telephone, 9.3\% through e-mail and $8.9 \%$ via QQ.

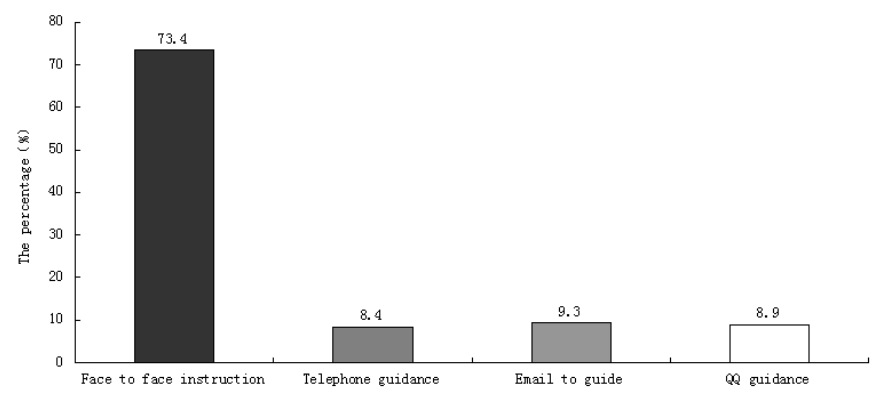

Fig.4 the means of instruction and communication

\section{b. instructor duties}

Instructors should strengthen the guidance toward students, and should make enough time to meet directly with students. At a critical time in graduation project progress, the instructors should not only play a role in the guidance of checks, and let go boldly in particular details, but also should give full play to the initiative and creativity of students. Instructors should continue to learn the leading trend of the industry, to encourage students to conduct innovative, challenging study, and to stimulate interest of the student. After the deciding the topic, instructors should encourage students to a broadly understand the relevant information, so as to further their own research ideas in some way; instructors should promptly check the progress, and put forward their own views and ideas according the results of the study, communicate with students and promptly point out the problems founded, in order to make the thesis written work to be carried out smoothly, instruct students to complete thesis later, amend the details, and guide students to complete reply.

\subsection{Students}

a. graduate project (thesis) difficulty faced by students

As is shown in Fig.5, for all the difficulties which is faced by students in graduate project (thesis), students think that mainly include impact from employment, entering a higher school, whether their accumulated knowledge is enough, or the condition of the experiment and books and materials are adequate, etc. It is not difficult to see that at current employment and postgraduate graduation exam is still the relatively prominent contradictions, so school should intensify the work of employment and build a platform to facilitate employment. In addition, facing of a large contingent of students, the work to constantly improve the experimental conditions, to increase the number of devices or further amplification experiments and research should be performed, in order to provide a more favorable hardware environment for the graduate project (thesis).

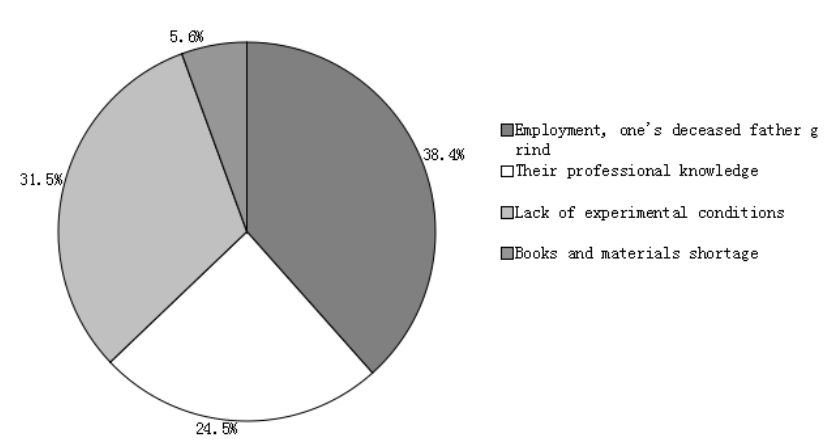

Fig.5 the main difficulties faced by students

b. the workload

An important indicator of whether the workload is saturated depends on the length of the thesis and the number of the read literature. "the undergraduate thesis (design) written standard of Jiamusi University " require that graduation project (thesis) should have about 10,000 words in length (excluding charts, formulas, procedures and calculations figure), and the amount of references should not less than 15. Fig.6 give the survey result about the length of the thesis and the references. It can be seen that most of the students have met the requirements of the workload of words and references, but there are still some students who do not meet the requirements referring to the literature. Literature is actually the first step in innovative research work, and plays a very important role.

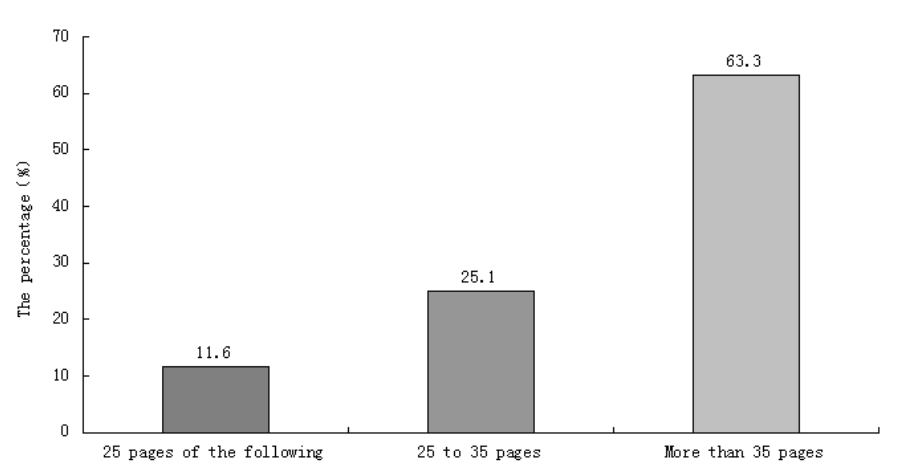

Fig.6 the length of the thesis

\section{c. students gains}

The purpose of graduation project (thesis) is to develop an ability of using basic theory, professional knowledge and basic skills to analyze and solve practical problems, so that students can primarily master the basic method of research. The 
development of independent working ability of students, independent thinking ability and also the ability to use knowledge to solve practical problems could be developed, especially the ability to independently acquire new knowledge. According to the investigation and analysis, $57 \%$ of students think that through graduate project, the integrated skills could be improved and strengthened, while $28 \%$ of students believe that network access or special training of graduate project meet the requirements for the graduation project, and the remaining $15 \%$ of the students think that the project are simply repeated past curriculum design, are consolidate the knowledge of what they have learned in the past, and failed to achieve the expected graduation purposes. Contact with the knowledge survey found that the design shown in by graduation, $72.4 \%$ of students believe that not only the textbook knowledge is used, but also master the new knowledge and has been used; $16.7 \%$ of the students thinks that only the textbook knowledge is used, while the rest of the students cannot answer this question clearly.

\subsection{Thesis process}

Graduation Project (Thesis) process arrangement has two main phases, the first phase is graduation project (thesis) subject research, literature reading and thesis proposal etc. Firstly topics should be chosen, and then issue written "Graduation Project (Thesis) mission statement," ,later propose the request about the subject, determine that the students has complete the thesis proposal, graduation project (thesis) working hours and working schedule. The second phase include thesis proposal, the implementation of graduate project (thesis), mid-term examination, thesis review, thesis reply work. Two stages of each part has its own time node, the result of the survey show that more than $80 \%$ of students can carry out in accordance with the time required for thesis work each part of each stage.

\section{CONCLUSION}

Through the analysis of the survey results, the profession being investigated can follow the latest regulation of the graduate project (thesis) management document to conduct management and organization work such as topics decision, tasks assignment, thesis proposal, mid-term examination, graduation project (thesis) guidance, thesis review and thesis reply work. But there are also shortcomings and problems, the following aspects and measures should be taken to improve the graduation project (thesis) management grade and thesis quality, so that the quality of the thesis could reach a higher level.

(1) Further expand the topics of graduate project (thesis), and enhance the usability and novelty of the topics.

(2) Take effective measures to ensure sufficient guidance and communication between instructors and students. An instructor should promote their own responsibility and ensure the quality and time of the guidance.

(3) Further enhance the management of the students. Special management, inspection supervision and guidance should be given to the students who has a slow progress and a poor quality.

(4) Strictly implement the quality management regulation and time node, and ensure a proper thesis progress, consequently the thesis could be accomplished with great quality and quantity.

\section{ACKNOWLEDGEMENTS}

This work was supported by Higher Education Scientific Research Projects of Heilongjiang Province (14G135, 14Z023); Jiamusi University Teaching Research Projects (JYLB2013-01, JYLA2012-007);

Jiamusi University Science Research Project (Lzp2012-03) and Jiamusi University Science and Technology Innovation Team Building Program (Cxtd-2013-01).

\section{REFERENCES}

[1] CAI Lili. Analysis and Countermeasure on Factors Affecting the Graduation Design. sQuality. Jou rna 1 of $\mathrm{H}$ enan Institute of Education (Natural Science), 2007,16(1): 36-38.

[2] WU Qiguang, Wang Liqiong. Survey and Analysis of Graduation Thesis. Science and education guides, 2012(3):121- 123.

[3] ZHANG Jing,Yao ji-tao,WU Fu-quan.An Analysis on Questionnalre about the Grakuation Project(Thesis). J.Xian Univ. of Arch. \& Tech.(Social Science Edition) 2011, 30(4): 80-83. 